\title{
Médiévales
}

Langues, Textes, Histoire

77 | automne 2019

Mathématiques. Savoirs et enseignements (OrientOccident)

\section{Anne CONCHON, Hélène NoIZET, Michel Ollion (dir.), Les Limites de Paris : XII ${ }^{e}-\mathrm{XVIII}{ }^{e}$ siècles}

Villeneuve d'Ascq, Presses Universitaires du Septentrion (« Histoire et civilisations »), 2017, $154 \mathrm{p}$.

\section{Stéphanie Pirez-Huart}

\section{(2) OpenEdition}

\section{Journals}

Édition électronique

URL : https://journals.openedition.org/medievales/10654

DOI : 10.4000/medievales. 10654

ISSN : $1777-5892$

\section{Éditeur}

Presses universitaires de Vincennes

\section{Édition imprimée}

Date de publication : 5 décembre 2019

Pagination : 183-185

ISBN : 978-2-37924-061-4

ISSN : 0751-2708

Référence électronique

Stéphanie Pirez-Huart, «Anne Conchon, Hélène Noizet, Michel Oluıon (dir.), Les Limites de Paris : xII ${ }^{e}$ xvIII siècles », Médiévales [En ligne], 77 | automne 2019, mis en ligne le 12 mai 2020, consulté le 22 avril 2022. URL : http://journals.openedition.org/medievales/10654; DOI : https://doi.org/10.4000/ medievales.10654 
Anne Conchon, Hélène Noizet, Michel Ollion (dir.), Les Limites de Paris : XII ${ }^{e}$ XVIII siècles, Villeneuve d'Ascq, Presses Universitaires du Septentrion (« Histoire et civilisations »), 2017, 154 p.

Cet ouvrage résulte d'une journée d'étude tenue en 2015 aux Archives nationales et inscrite dans le cadre du projet du Grand Paris. Le but des organisateurs était d'étudier les fonctions et les usages des limites de la ville, aussi bien en tant que marqueur de différents espaces que comme vecteur d'interaction à l'intérieur du territoire urbain. L'ouvrage pose donc la question des limites physiques, « morales », juridiques et du rapport des hommes du temps à ces limites et aux pratiques qui en découlent. Dans son introduction, Anne Conchon rappelle que l'étude des limites est ancienne chez les historiens et cite notamment le travail de Michel Deveze sur la banlieue (p. 11). L'étude des limites de Paris est à envisager sous deux angles. La limite est d'abord physique et fait de la ville un espace clos, par exemple à travers les enceintes militaires d'une part et les bornes d'autre part, installées par le pouvoir royal conjointement à un recensement des maisons des faubourgs au XVIII ${ }^{\mathrm{e}}$ siècle pour freiner l'urbanisation. La limite est également imaginaire, par exemple lorsqu'il est question des divisions administratives et sociales. Accessibles en particulier à travers les vestiges et les représentations cartographiques, les limites et leur étude sont indispensables à la compréhension du changement social et de la transformation physique de la ville ; elles déplacent le regard du chercheur de la vision unifiante du tissu urbain à une perspective fragmentée et ouvrent la voie à une meilleure appréhension de l'aire d'influence de la cité. Sept articles et une conclusion d'Anne Conchon et Hélène Noizet tentent donc d'éclairer ce thème, suivant une démarche transpériodique qui court du XII ${ }^{\mathrm{e}}$ siècle jusqu'au XVIII ${ }^{\mathrm{e}}$ siècle, dans une perspective interdisciplinaire soulignée dès l'introduction (p. 11-12).

Michel Ollion souligne l'impression de dispersion des sources qui se dégage du travail de Marcel Poete et lui substitue un panorama des sources utiles à l'étude des limites, en adoptant l'angle des institutions susceptibles de produire les documents intéressant l'historien. Son étude montre que les limites constituent un thème qui préoccupe les autorités sous l'Ancien Régime, qui créent en 1775 un bureau topographique pour la démarcation des limites auprès du secrétariat d'État aux affaires étrangères. Il met également en évidence la multiplicité des acteurs qui interviennent et développe l'exemple de la déclaration royale de 1724 au sujet du bornage : Conseil du roi, Parlement de Paris, bureau de la ville de Paris, bureau des finances de la généralité de Paris. Outre les autorités publiques qui interviennent pour édicter des règlements ou prennent en charge la construction et l'entretien des remparts, il insiste sur les acteurs qui bénéficient de privilèges ou font l'objet d'obligations découlant de ces limites. Cela induit une diversité des sources exploitables par le chercheur : sources royales et seigneuriales, mais aussi archives fiscales, judiciaires ou notariales par exemple, tandis que les sources littéraires restent marginales sur ce sujet.

L'article écrit conjointement par Hélène Noizet et Caroline Bourlet se démarque des autres contributions. En dépit de la longueur de l'exposé, la démonstration qu'elles réalisent est claire et bien argumentée. Elles livrent ici les résultats d'une enquête visant à définir la banlieue parisienne à travers plusieurs siècles, couvrant les époques médiévale et moderne. Réfutant l'étude d'A. Jourdan, elles démontrent comment on passe d'une banlieue conçue d'abord dans son aspect juridique à un espace davantage envisagé dans son versant géographique à la fin de l'Ancien Régime. Les auteures, compilant les informations de plusieurs sources qui se recoupent, concluent à une banlieue qui s'étend sur un rayon resserré, en majorité entre cinq et dix kilomètres du Châtelet, faisant de Paris une exception au regard des travaux de Bernard Chevalier sur le sujet. Cette recherche se fonde à la fois sur des sources textuelles et cartographiques : le propos est 
d'ailleurs richement illustré par de nombreuses cartes, pour lesquelles on regrette toutefois l'impression en noir et blanc qui rend la lecture parfois malaisée.

Pierre-Henri Guittonneau s'attache à déterminer la place occupée par les Parisiens dans les villes principales de la région parisienne - essentiellement situées sur la Seine ou ses affluents - à travers les transactions enregistrées dans les minutes des notaires du Châtelet à la fin du $\mathrm{Xv}^{\mathrm{e}}$ siècle. Il confirme l'organisation géographique de la région parisienne mise en évidence en 1964 par Guy Fourquin et montre que les habitants de la capitale font des affaires au plus près d'eux, en particulier dans les villes participant à l'approvisionnement de Paris. Ce lien avec l'économie est d'ailleurs perceptible dans la nature des sources utilisées pour cette étude ainsi que dans le profil des contractants, puisqu'il s'agit essentiellement de marchands, qu'ils soient bourgeois ou artisans, puis d'officiers royaux. Les Parisiens confortent ainsi leur fortune préalablement établie. L'auteur remarque que, si la renommée et l'honneur constituent des facteurs déterminants dans le choix des partenaires, la question de la parenté est également importante, puisqu'elle concerne un quart des transactions étudiées. Enfin, en ajoutant Mantes-laJolie au corpus - elle était exclue par Guy Fourquin -, il met en évidence le localisme des contractants et la primauté des Parisiens et des Normands (dans une moindre mesure), parmi les partenaires.

Nicolas Lyon-Caen s'attache à l'étude des barrières fiscales au XVIII ${ }^{\mathrm{e}}$ siècle, avant la construction du mur des Fermiers généraux. Il montre que ces points de perception désignés dans les sources sous des termes variés visent à la fois le contrôle administratif et le prélèvement des taxes. Les barrières sont des éléments du paysage, essentiellement bâties en bois, qui permettent parfois de loger les commis, bien que ces derniers soient majoritairement hébergés chez des particuliers. Elles sont un point de convergence des marchandises, mais génèrent également des déplacements depuis l'intérieur de la ville, à l'exemple des destinataires qui y viennent pour régler directement les taxes. Enfin, l'auteur montre aussi en quoi elles assurent une jonction avec les espaces environnants de la ville, tout en soulignant qu'elles ne marquent pas la limite de la ville, mais qu'elles sont bien incluses à l'intérieur du territoire urbain.

Mathieu Marraud montre l'opposition entre les marchands et les artisans à travers l'usage des termes désignant les structures juridiques les réglementant (corps/communauté). Cette dichotomie lui permet de déterminer comment cette hiérarchie qui s'installe entre les deux groupes sociaux se traduit dans l'occupation de l'espace urbain. L'opposition qui en résulte s'observe également dans les litiges étudiés par l'auteur.

Nicolas Vidoni porte la focale sur la façon dont, à la fin de l'époque moderne, les limites juridictionnelles organisent le travail de la police et comment cette dernière doit les franchir pour tenir compte des réalités sociales. Il démontre que l'extension de la ville est redoutée par les forces de l'ordre en raison de l'étendue de territoire à maitriser. Mais la ville croît, malgré les tentatives de la monarchie pour y mettre un frein. L'auteur explique que le changement des limites qui en découle nécessite le recours à des auxiliaires de police et à une collaboration avec d'autres forces mobilisées. En dépit de l'extension du tissu urbain, la mobilité de la police continue d'augmenter, et elle est régulièrement contrainte de se rendre dans les faubourgs, par exemple pour traquer les voleurs en période de foire.

Enfin, Diane Roussel s'interroge sur la façon dont les Parisiens perçoivent et vivent la limite de la ville au $\mathrm{XVI}^{\mathrm{e}}$ siècle, en s'appuyant notamment sur les archives criminelles. En étudiant le quartier de Saint-Germain-des-Prés, elle démontre comment l'intégration d'un espace à l'intérieur de la ville vise à chasser les criminels, par exemple à travers les horaires d'ouverture des portes, plus ou moins bien respectés. In fine, c'est le supposé ordre urbain qui est recherché par ceux qui résident à la limite de la ville. 
Anne Conchon et Hélène Noizet concluent à une divergence des limites selon le prisme d'observation. Elles soulignent également combien ces lignes, physiques, administratives, sociales sont des constructions historiques et mouvantes. L'ouvrage pose donc des bases solides à l'étude des limites de Paris, et des villes de façon plus générale, et invite à poursuivre l'enquête, notamment en changeant d'échelle d'étude.

Stéphanie PIREz-Huart (Université Polytechnique des Hauts-de-France, CALHISTE

Marie-Laure DeRAT, L'Énigme d'une dynastie sainte et usurpatrice dans le royaume chrétien d'Éthiopie du XI au XIII siècle, Turnhout, Brepols (« Hagiologia », 14), 2018, $329 \mathrm{p}$.

L'histoire de l'Afrique ne se limite pas à des allusions malheureuses dans des discours politiques, des indignations autour de son insertion puis de son retrait dans les programmes du collège ou des querelles à propos de la création d'une chaire au collège de France. Moins visible dans le débat public, l'historiographie française participe activement au renouvellement des savoirs sur l'Éthiopie ancienne. Connue notamment pour son travail issu de sa thèse de doctorat - sur la construction territoriale du pouvoir royal éthiopien aux $\mathrm{XIV}^{\mathrm{e}}{\text { et } \mathrm{XV}^{\mathrm{e}} \text { siècles }}^{6}$, Marie-Laure Derat s'inscrit dans cette tradition et contribue à sa vitalité. Issu de son habilitation à diriger des recherches, cet ouvrage est la première véritable synthèse sur les Zagwé, dynastie qui régna sur l'Éthiopie entre le $\mathrm{XI}^{\mathrm{e}}$ et le $\mathrm{XIII}^{\mathrm{e}}$ siècle, connue par les églises qu'elle fit creuser dans le roc sur le site de Lalibala et présentée dans tous les travaux sous un double jour : une dynastie usurpatrice, parenthèse entre les souverains d'Axoum et la dynastie salomonienne, et une dynastie sainte, exaltée par un large corpus hagiographique.

Afin de résoudre ce paradoxe, l'ouvrage revient aux bases de la recherche historique : étudier la dynastie à partir des textes qui lui sont contemporains et cantonner la tradition postérieure à l'analyse de sa mémoire. Cette méthode de bon sens n'avait jamais été mise en œuvre, et pour cause : les sources contemporaines des Zagwé, publiées et présentées en détail dans la première partie, se limitent à quatre donations, une note de consécration d'une église et dix dédicaces de meubles d'autel ou croix votives. Même en y ajoutant les apports de l'archéologie - en particulier le site de Lalibala, où l'auteure codirige un projet de fouilles depuis 2009 - et les textes produits en Égypte (deux chroniques et une lettre), l'ensemble se prête difficilement à l'exercice de la monographie dynastique. Les deux parties centrales étudient pourtant les origines et les premiers temps de cette dynastie, puis son organisation à l'époque de son souverain le mieux connu, Lalibala, qui fit creuser l'essentiel des églises sur le site du même nom. On comprend aisément qu'elles sont principalement constituées d'hypothèses, élaborées à partir d'indices ténus par une analyse soigneuse des rares documents, leurs comparaisons et leurs croisements. En étudiant séparément dans une dernière partie tous les récits rédigés sur les Zagwé depuis leur chute vers 1270 jusqu'à l'historiographie contemporaine, l'ouvrage révèle le hiatus profond existant entre ce qu'il est possible d'affirmer sur cette dynastie et ce qui en a été dit jusqu'à aujourd'hui.

Le travail de Marie-Laure Derat propose donc une révision presque complète de toutes les connaissances considérées acquises sur les Zagwé. Il serait pour cela illusoire de vouloir en présenter tous les apports. On se limitera à souligner certains points, en ayant conscience du caractère arbitraire de cette sélection, propre aux intérêts et attentes de l'auteur du présent compte rendu.

6. M.-L. Derat, Le Domaine des rois éthiopiens (1270-1527). Espace, pouvoir et monachisme, Paris, 2003. 